\title{
1 A comparison of the sensory and rheological properties of different cellulosic
}

2 fibres for food

3

4 Division of Food Sciences, School of Biosciences, University of Nottingham, Sutton

5 Bonington Campus, Loughborough, LE12 5RD, UK.

$6 \quad *$ Corresponding author e-mail: tim.foster@nottingham.ac.uk

\section{Abstract}

8 The impact of different cellulosic microstructures formed by highly entangled fibre networks 9 were studied for food applications as dietary fibre. This paper reports the impact of microstructure on the rheological and sensory behaviour of the aqueous suspensions of particulate and fibrillated forms of softwood cellulosic fibres, and were compared with citrus fibre. An aqueous suspension of cellulosic fibres shows stable viscoelastic gel-like behaviour as a function of frequency. The particulate form of cellulosic fibres showed lowest shear viscosity as compared to the entangled network system at comparable concentrations. To provide further insight into the relationship between the structure of cellulosic fibre systems and perception of salt taste in aqueous suspensions of softwood cellulosic fibres (fibrillated and particulate form) and citrus fibres with matched shear viscosities were studied. A hypothesis to explain why softwood cellulosic fibre (CTE) with entangled network structure prolongs the taste perception is presented.

\section{Introduction}

Polysaccharides are known to be used as functional ingredients in a wide range of commercial applications such as food, personal care and pharmaceutical products. In the food industry, polysaccharides are used as thickening, gelling, emulsifying, stabilisation and coating agents [1]. For these purposes, different natural polysaccharides such as starch, carrageenan, guar gums and bacterial polysaccharides such as xanthan and bacterial cellulose are used. Typically, a combination of two or more of these hydrocolloids is used to create a variety of 
microstructures to formulate stable food applications with specific attributes like acceptable mouthfeel and flavour perception. The processing conditions such as shearing, heating and pumping of the polysaccharide mixtures result in microstructures with unique rheological and sensory properties such as fat mimicking, and texture enhancement [2,3,4]. These polysaccharides are typically categorised as dietary fibres in the food and pharmaceutical industry. By definition, according to American Association of Cereal Chemists in 2000, dietary fibres are referred to as the edible parts of plants or analogous carbohydrates that are resistant to digestion and absorption in the human small intestine with complete or partial fermentation in the large intestine [5]. Fibres are often classified as soluble dietary fibre and insoluble dietary fibre [6]. These dietary fibres may consist of non-digestible carbohydrates, cellulose and lignin that are an intrinsic part of a plant cell wall [7]. Depending on the source of fibres the amount of soluble and insoluble components vary, for instance, the dietary fibre from fruits and vegetables contains considerably higher proportion of soluble fibres, whereas cereal, peel of fruits or other crops contain more insoluble components such as cellulose and hemicellulose [8]. Dietary fibres play an important role in human health, as it was reported in past that high dietary fibre diets are associated with the prevention, reduction and treatment of some diseases, such as reducing cholesterol and maintain gastrointestinal health $[9,6]$.

The dietary fibre produced from citrus fruit such as CitriFi and Herbacel AQ+ are widely used in various dairy products as a fat replacer, in low-fat mayonnaise, salad dressing and icecreams, providing fibre frameworks to improve water-holding capacity and therefore acting as a thickening agent. Whereas in bakery products such as biscuits, croissants and muffins etc. these fibres are used as fat and calorie reducing agents without compromising taste, texture and cost $[8,10]$. The rheological parameters such as flow behaviour and viscoelastic behaviour of the different food applications or model systems can be correlated with the sensory texture properties and stability of the products $[11,12]$. Depending on the source, type and concentration of fibres used in the product, different rheological and textural properties can be 
achieved. For instance, the presence of xanthan gum improves the texture and physical shelflife of oil-in-water emulsions such as a salad dressing. The citrus fibre in combination with another stabiliser such as xanthan or LBG improve the physical, chemical and sensory properties of ice-cream samples [13, 14]. Similarly, a number of other cellulosic fibres are used in the food industry such as MCC (microcrystalline cellulose), CMC (carboxymethyl cellulose) and the other chemically derivatised celluloses such as methylcellulose.

Rheologically, a number of studies showed that an aqueous suspension of citrus fibres and cellulosic fibres such as MCC or MFC (microfibrillar celluloses) shows gel-like behaviour where the storage modulus $\left(G^{\prime}\right)$ is higher than the loss modulus ( $\left.G^{\prime \prime}\right)$ over a wide concentration range. These moduli show little frequency dependence at all concentrations $[15,16,17,18$, 19]. The higher elastic modulus might be due to long fibrils and fibrillar-aggregates and entangled microfibrils, forming strong network structures. These aqueous suspensions of different cellulosic fibres also exhibit shear thinning behaviour [16, 20]. Similar rheological properties were observed by an aqueous suspension of dietary fibres extracted from tomato peel/pomace and date $[21,22]$. The objective of the current publication is to provide an insight into the rheological properties of microfibrillar cellulosic fibre extracted from softwood spruce and understand the fundamental differences between the different cellulosic fibre microstructures and their functionality in food.

In recent years, an increase in demand for the low-salt food products was reported by the food industry, due to continuous awareness from health professionals. Associations have been made between a high sodium diet and an increased risk of certain health conditions such as hypertension and cardiovascular disease [23, 24]. Hence, the World Health Organisation (WHO) recommendations for a daily salt intake limit of $5 \mathrm{~g}$, recognising that many consumers exceed this limit approx. > 10g [26]. However, salts play many important roles in food products not just as a tastant enhancing flavour, but also affecting physical properties, shelf-life and 
finally prevention of fermentation (Lynch et al., 2009). Different salt replacement work has been presented in the past such as in bread; salt $(\mathrm{NaCl})$ replaced with potassium or magnesium salts resulted in unpalatable metallic, bitter and off-taste [27]. Rama (2013) showed that the size of salt crystals influenced the rate of salt perception. It was reported that the larger salts crystals dissolved relatively slowly as compared to small salt crystals, this prolongs the duration of the taste perception. Ultimately, the smaller salt crystals meant less salt was required to achieve a similar level of salty taste [28]. It is well established that as the viscosity of the hydrocolloid thickened product increases, the flavour perception of the product decreases especially when the concentration of hydrocolloid exceeds the critical overlap concentration i.e. $\mathrm{c}^{*}[29,30,31]$. This decrease in flavour perception is due to a reduction in the amount of tastants reaching the sensing organs [32] due to an increase in viscosity of the system. Depending on the type of hydrocolloid used in the product a noticeable impact on flavour and taste perception is observed, for instance, the products thickened with starch showed good taste and flavour perception as compared to product thickened with xanthan $[33,34]$.

The primary aim of this study then is focused on understanding the impact of highly entangled networks of cellulosic fibre from various sources and their impact on rheological properties of the suspension. It is hoped that this understanding will shed light on the potential application of cellulosic fibres extracted from softwood (spruce) in food applications. A detailed study of microstructure was performed by using light microscopy and correlated with water retention capacity and rheological behaviour of the suspensions. The second objective of the work was to test the impact of the highly entangled network of cellulosic fibres on overall taste (sensory) perception from a basic food model system composed of cellulosic fibres, water and salt. A detailed study of rheological behaviour and the sensory perception of the cellulosic fibres, when correlated with light microscopy, as presented here, will enable important structural features of these cellulosic materials to be identified which are of relevance to the food and personal care industries alike. The hypothesis underpinning this research is that the highly 
entangled network microstructure of cellulosic fibres are responsible for higher water retention capacity which also reflects significantly on higher rheological properties and lowers the taste (sensory) perception.

\section{Materials and methods}

\subsection{Materials}

For this study different food grade, cellulosic fibres: citrus fibres CF100 and CFAQ+ were provided by Cybercolloids, Ltd (Ireland) and CTE (Flakes and Powder form, and are composed on softwood spruce cellulose (CTE) and carboxymethyl cellulose (CMC)) was provided by Borregaard AS (Norway). Reverse osmosis (RO) water was used for all sample preparation. Sensory data was collected using FIZZ 2.0 software (Biosystems, Couternon, France).

\subsection{Sample preparation}

All cellulosic fibres were dispersed in RO-water by using a high shear Ultra-turrax homogeniser at 18000rpm for 4 minutes at different concentrations (between $0.1 \%-2.5 \%$ w/w). All the samples were left to hydrate overnight on roller bed (60rpm speed) at ambient temperature before analysis. The concentration of all the sample was checked by using OHAUS MB25 moisture analyser (OHAUS, US). All samples were freshly prepared in two batches and analyses were made in duplicate. For sensory analysis different cellulosic fibres (i.e. CTE (flakes), CTE (Powder), CF100 and CFAQ+) were dispersed in $0.2 \% \mathrm{NaCl}$ stock solutions at different concentrations at comparable viscosities i.e. high (0.2Pas) and low (0.01Pas) at 50 $\mathrm{s}^{-1}$ shear rate (summarised in Table 1) and also at constant concentration $(1.5 \% \mathrm{w} / \mathrm{w})$. All samples were mixed by using a high shear mixer (Silverson, UK) at 5000rpm for 5mins. All samples were stored at $4^{\circ} \mathrm{C}$ overnight and stirred well before serving to panellists. For sensory analysis, all ingredients are commercially available and commonly used in a variety of food products. Prior to sensory evaluation, all panellists were informed of the ingredients and any possible allergens highlighted in accordance with local Sensory Centre procedures. 
Table 1: Different concentrations (Concn \%) of cellulosic fibres and corresponding shear

130 viscosity $\left(\right.$ at $\left.50 \mathrm{~s}^{-1}\right)$ used for sensory analysis.

\begin{tabular}{|l|c|c|c|c|}
\hline \multirow{2}{*}{ Sample } & \multicolumn{2}{|c|}{ High Viscosity } & \multicolumn{2}{c|}{ Low viscosity } \\
\cline { 2 - 5 } & Concn (\%) & Shear Viscosity (Pas) & Concn (\%) & Shear Viscosity (Pas) \\
\hline CTE (F) & 1 & 6.56 & 0.2 & 0.194 \\
\hline CTE (P) & 1.5 & 5.59 & 0.5 & 0.0918 \\
\hline CFAQ+ & 1 & 5.35 & 0.8 & 0.0812 \\
\hline CF100 & 2 & 7.45 & 0.5 & 0.0918 \\
\hline
\end{tabular}

131

\subsection{Rheological Analysis}

133

134

The rheological measurements were carried out on a stress-controlled Rheometer (Physica MCR 301, Anton Paar, Austria) with a serrated parallel plate $(50 \mathrm{~mm}$ diameter with a gap of $1 \mathrm{~mm})$ at $20 \pm 1^{\circ} \mathrm{C}$, controlled by a Peltier system. Small oscillation amplitude sweeps were generated by log ramping strain $0.01-100 \%$ at a constant frequency of $1 \mathrm{~Hz}$. Frequency sweeps were performed over the frequency range $0.1-15 \mathrm{~Hz}$ at a constant strain of $0.2 \%$ which lay within the linear viscoelastic region. Rotational measurements were performed by increasing the shear rate from $0.01-10001 / \mathrm{s} \log$. Data presented is an average of four replicates.

\subsection{Sensory evaluation}

Panellists ( $n=74$, aged 20-40, mixed male and female volunteers) were recruited from the University of Nottingham staff and students. The four samples (CTE (flakes), CTE (Powder), CF100 and CFAQ+) were compared for saltiness using a round robin of paired comparison (PC) tests (BS EN ISO 5495:2007), such that each sample was evaluated against every other sample within the set of four, ensuring a total of 6 paired comparison tests. Three separate sessions were performed to examine the saltiness perception at a low viscosity ( $0.1 \mathrm{Pas}$, Panellist: 74), high viscosity (6Pas, Panellist: 74) and at matched fibre concentration (1.5\% w/w, Panellist: 60). The sample size was $10 \mathrm{ml}$ throughout and samples were served at room temperature $\left(20 \pm 1^{\circ} \mathrm{C}\right)$. For each test the panellist had to take the whole sample in their mouth, 
allow the sample to coat the roof of their mouth, hold in the mouth for a minimum of 5 seconds before swallowing, and then cleanse their palate with unsalted crackers (99\% Fat Free, Rakusen's, Leeds, UK) and mineral water (Evian, France) before tasting the next sample. Panellists were instructed to determine which of the 2 samples was highest in 'saltiness'. Rest breaks were given between every 3 paired comparison tests. The test was used in forced-choice mode, so panellists were required to give an answer even if the perceived difference was negligible. Panellists were asked to provide additional comments regarding any other differences between the samples. All tests were carried out at the University of Nottingham's Sensory Science Centre, within individual sensory booths under controlled temperature and humidity. Testing was performed under red light in order to minimise any small differences in sample colour not relevant to the test. All the experiments were performed in compliance with UK legislation (ISO standards), and in accordance with the institutional framework and practices established by the University of Nottingham Ethics Committee. All participants received written information about the study before giving their informed consent.

\subsection{Microscopic analysis}

Light microscopy of all aqueous suspensions of samples was performed by using Olympus BX5 bright field light microscopy at 20X magnification, scale bar $200 \mu \mathrm{ms}$, all fibres were dyed using Congo red dye (Sigma-Aldrich, UK).

\subsection{Water Retention Values (WRV):}

Approximately $0.1 \mathrm{~g}$ (A) of powder was added to $100 \mathrm{~g}$ water and mixed with an Ultra-turrax for $4 \mathrm{mins}$ at $18000 \mathrm{rpm}$. The mixture was placed in a centrifuge tube and allowed to rest for 2hrs followed by centrifugation (Beckman Centrifuge machine, Model: J2-21) for 30mins at 2141g. The top water layer was removed and the bottom layer weighed (B). This was done in duplicate, and WRV was calculated by using Equation 1 (Eq.1). 
Calculation: WRV $(\%)=($ Bottom layer $(\mathrm{B})$-starting material (A) $) /$ starting material (A) Eq. 1

175

176

177

\subsection{Data Analysis}

Sensory data were collected by using FIZZ 2.0 sensory software (Biosystems, Couternon, France) and statistical analysis was performed by using Friedmans approach (significant level $\alpha=0.05)$. Rheology and WRV data were analysed by using ANOVA. The main purpose of the ANOVA test is to identify and quantify the factors which are responsible for the variability of the response.

\section{Results and Discussion}

\subsection{Microstructure of cellulosic fibres:}

Light microscopy images of different cellulosic dietary fibres at $1.5 \% \mathrm{w} / \mathrm{w}$ concentration are presented in Figure 1. A noticeable difference in the microstructure was observed while comparing $\mathrm{CTE}(\mathrm{F})$ or $\mathrm{CTE}(\mathrm{P})$ with the citrus fibres $\mathrm{CF} 100$ and $\mathrm{CFAQ}+$. The aqueous suspension of CTE $(\mathrm{F})$ showed a dense entangled fibre network whereas larger aggregates and fibre bundles were observed with $\mathrm{CTE}(\mathrm{P})$. CTE(P) samples were produced by further milling process of $\mathrm{CTE}(\mathrm{F})$ product. During the milling process, the system exhibits slight moisture loss, hence fibres form strong intermolecular interactions (common phenomena known for cellulosic fibres upon drying or moisture loss with an increase in temperature), which explains the noticeably higher amount of fibre aggregates upon hydration. The aqueous suspension of citrus fibres i.e. CF100 and CFAQ+ showed multiple components (both soluble and insoluble) in the system such as short fibre bundles of fibre, globular structures which are believed to be pectin and other cell wall material (similar microstructures were observed by Córdoba et al., 2010 with lemon fibres). Larger cellulosic fibre bundles and noticeably less interconnected fibre-network were observed in the case of both CFAQ+ and CF100 (Figure 1). These highly entangled fibre network microstructures are responsible for the noticeable difference in both 
201

202

204

205

206

water retention value (also known as water retention capacity) and rheological properties of the suspensions, discussed below.
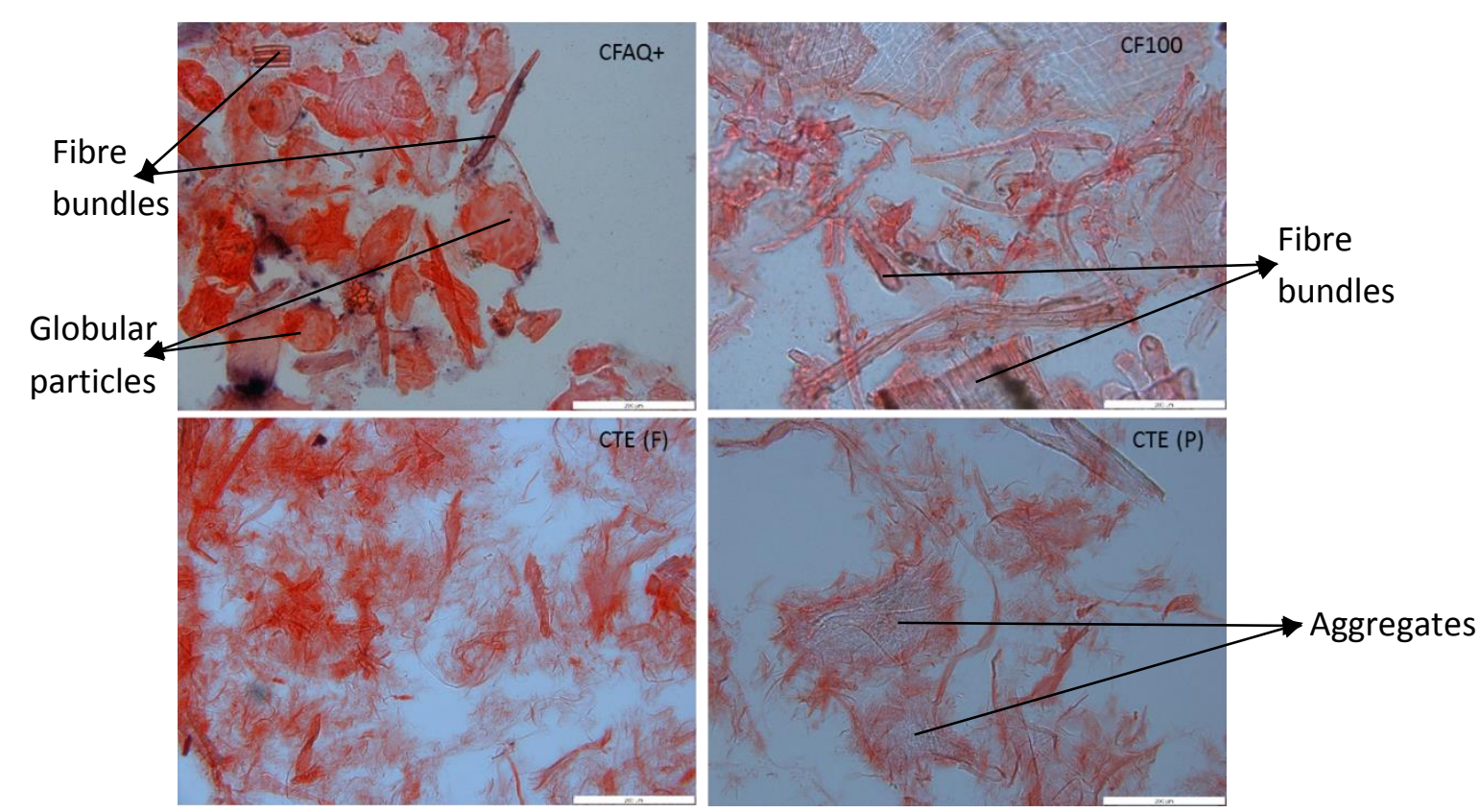

Figure 1: Light microscopy images of 1.5\% aqueous suspension of different cellulosic fibres stained with Congo red dye, scale bar: $200 \mu \mathrm{ms.}$

Water retention value (WRV) is an important property of dietary fibres from both a physiological and technological point of view. It helps in understanding the behaviour of dietary fibres in food applications or during gut transit. WRV of the different cellulosic fibres are presented in Figure 2, a significant difference ( $p$-value<0.05) in WRV was observed when comparing the softwood cellulosic fibre $(\mathrm{CTE}(\mathrm{F})$ and $\mathrm{CTE}(\mathrm{P}))$ and citrus fibres $(\mathrm{CF} 100$ and CFAQ+). This significant difference in the WRV can be explained by the different microstructures, inherent formulation (soluble and insoluble components present in the system) and different processing. It is well established that the processes such as drying, grinding, heating or extrusion process modifies the physical properties of the fibre matrix and hydration properties [35, 36]. Sangnark \& Noomhorm (2003) and Elleuch (2011) reported that the grinding can damage the regions of potential water retention capacity and, therefore, decrease the capacity to hold water $[37,38]$. This explains why lower amounts of water were retained 
221 in the CTE(P) fibre-network (milled product) whereas higher amounts retained in $\mathrm{CTE}(\mathrm{F})$

222 network structures. A slight loss of moisture during the process might have introduced different

223 intermolecular interactions, resulting in larger amount of aggregates (evident in Figure 1), these

224 aggregates are difficult to hydrate hence reducing the water retention capacity. Whereas lower

225 WRV of citrus fibres suspensions can be explained by larger fibre bundles, a noticeably less

226 interconnected fibre network and the presence of other soluble and insoluble components

227 present in the case of CFAQ+ and CF100 (Figure 2 and Figure 1; similar behaviour with other citrus fibres was reported by Grigelmo-Miguel et al., 1999 [39]). Interestingly, the WRV of CFAQ+ was higher than CF100 this can be explained by two factors i.e. (1) inherent differences in the soluble and insoluble content in the formulation, and (2) entangled network structure

231 formed during the processing of these fibres. The difference due to the formulation correlate 232 well with WRV reported with orange dietary fibres [39], lime peel [40], mango dietary fibre 233 [41], peach dietary fibre [42] and carrot dietary fibre [43]. Also, it is evident from the 234 microstructure of citrus fibres in Figure 1, that the CFAQ+ has slightly smaller fibre size and 235 is much more entangled than CF100, hence affecting the water retention capacity of the fibres. 236 It is well established that the hydration and water retention capacity of dietary fibres are very 237 important factors in the food industry as these factors can influence the ingredients functionality, shelf life and product yield $[44,45]$. The high WRV of CTE(F) suspension 
239 240 like the industry established citrus fibres.

241

242

243

244

245

246

247

248

249

250

251

252

253

254

255

256

257

suggested that the material could be used as a functional ingredient in food applications just

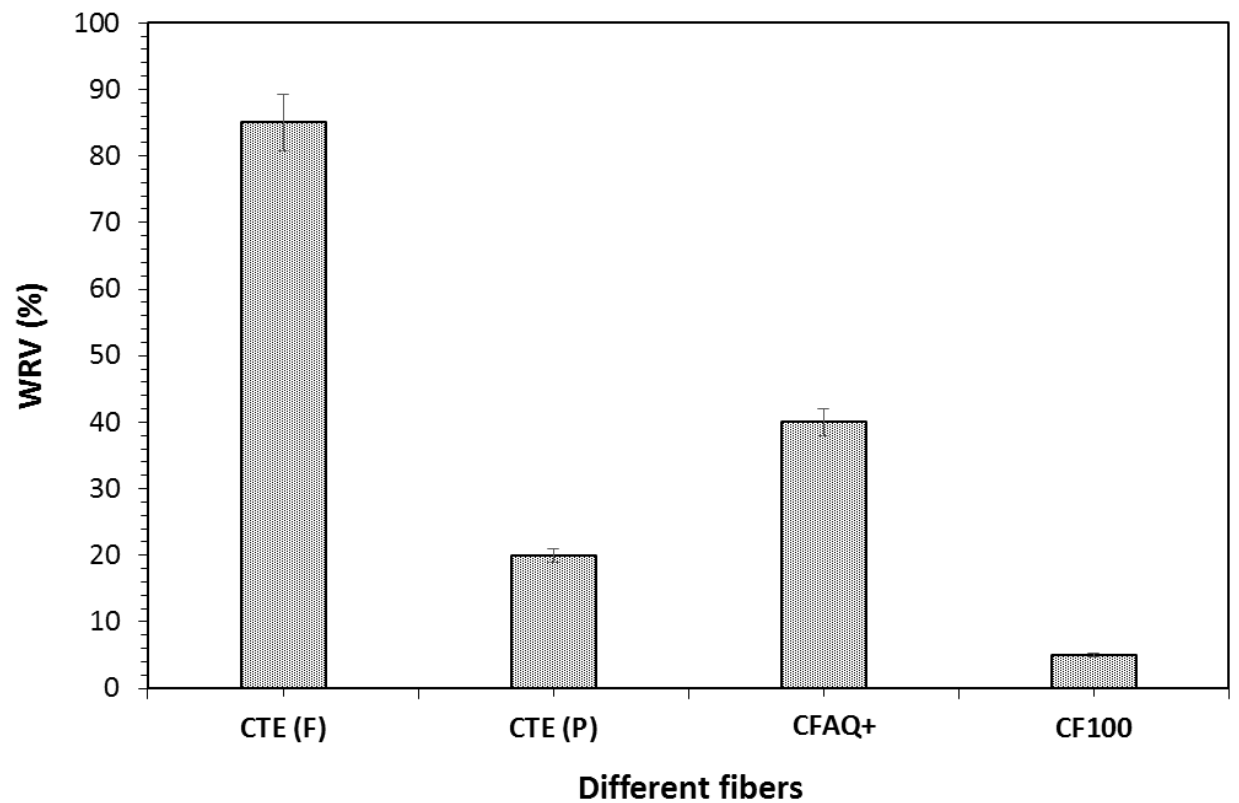

Figure 2: Water retention values (WRV \%) of different cellulosic fibres.

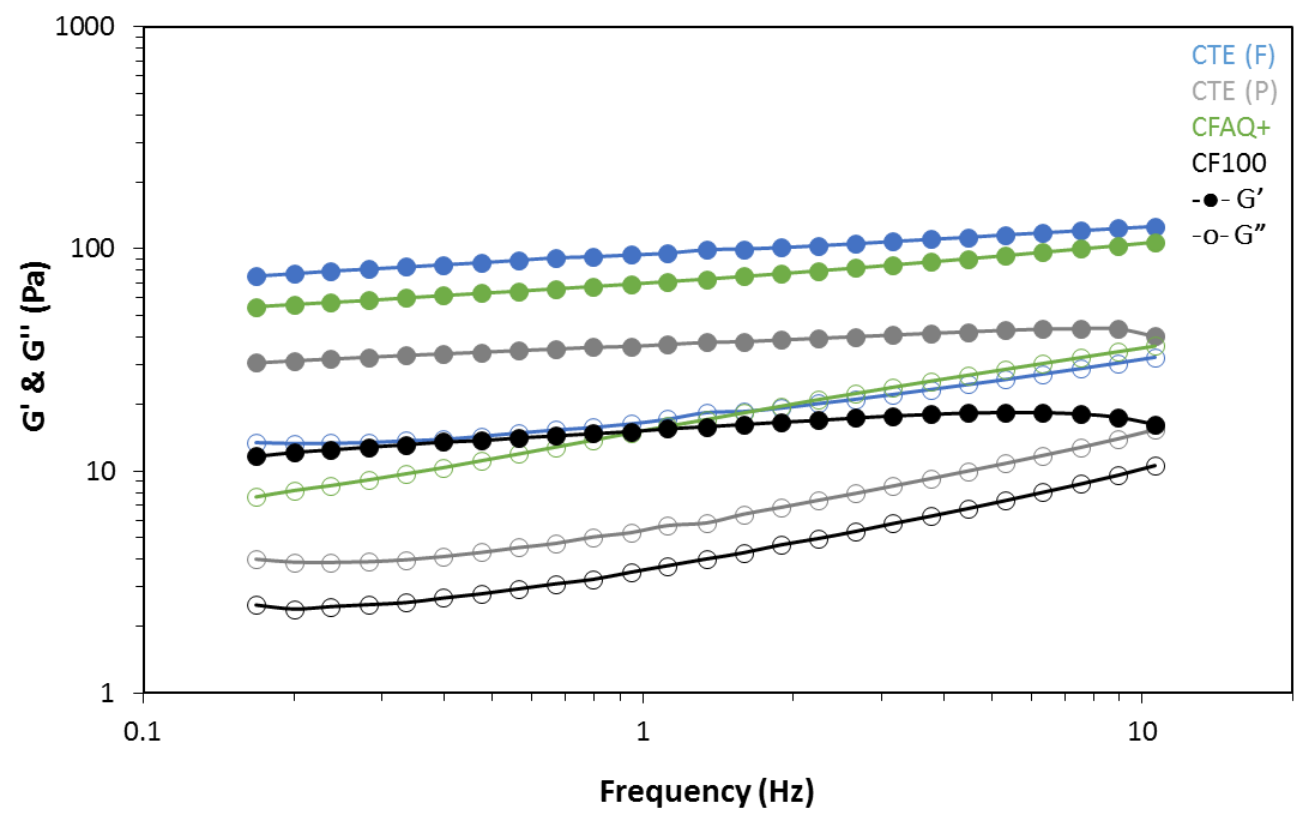

Figure 3: Dynamic mechanical spectra of $1.5 \%$ w/w aqueous suspension of $C T E(F), C T E(P)$ and citrus fibres i.e. CFAQ+, CF100, where storage modulus (solid symbols) and loss modulus values (unfilled symbols) are represented as a function of frequency measured at $20 \pm 1^{\circ} \mathrm{C}$.

\subsection{Rheological properties of cellulosic suspensions}


260 Storage modulus $\left(G^{\prime}\right)$ and loss modulus $\left(G^{\prime \prime}\right)$ as a function of the frequency of an aqueous 261 suspension of CTE ( F and P) and citrus fibres i.e. CFAQ+ and CF100 are presented in Figure

262 3. All suspensions showed viscoelastic gel-like behaviour, where storage modulus was higher than loss modulus with little dependency on frequency. Similar behaviour was observed with lemon fibres by Cordabo et al., 2010 [17] and softwood cellulosic fibres by Tatsumi et al., 2007 [19]. Slight dependency of G' \& G” on the frequency indicates that the network structure formed by cellulosic fibres (independent of source) is in an active mode of forming entanglements to form a stable network of fibres, producing a suspension with gel-like properties. Chen (2013) suggested that high frequency increases the mobility of microfibers in aqueous suspension, this increased mobility of the microfibers results in increases the entanglement and formation of densely ordered network structure which reflects on viscoelastic behaviour [46] . At a comparable concentration of $1.5 \% \mathrm{w} / \mathrm{w}$, the elastic moduli of CTE(F) was

272 highest, where $\mathrm{CTE}(\mathrm{F})>\mathrm{CFAQ}+>\mathrm{CTE}(\mathrm{P})>\mathrm{CF} 100$, following the same trend for WRV (Figure $2)$, and visually explained when considering the highly entangled network of the $\mathrm{CTE}(\mathrm{F})$ aqueous suspension (Figure 1). The aqueous suspension of CF100 showed the lowest moduli, WRV values and have relatively large and discrete fibre particulates in the matrix, which explains the weak viscoelastic behaviour of the suspension. Whereas, CTE(F) flakes show

277 higher moduli when compared to $\mathrm{CTE}(\mathrm{P})$ powder form, and can be explained by the retention 278 of a more fibrillated structure (Figure 1) resulting in higher water retention values (Figure 2). 


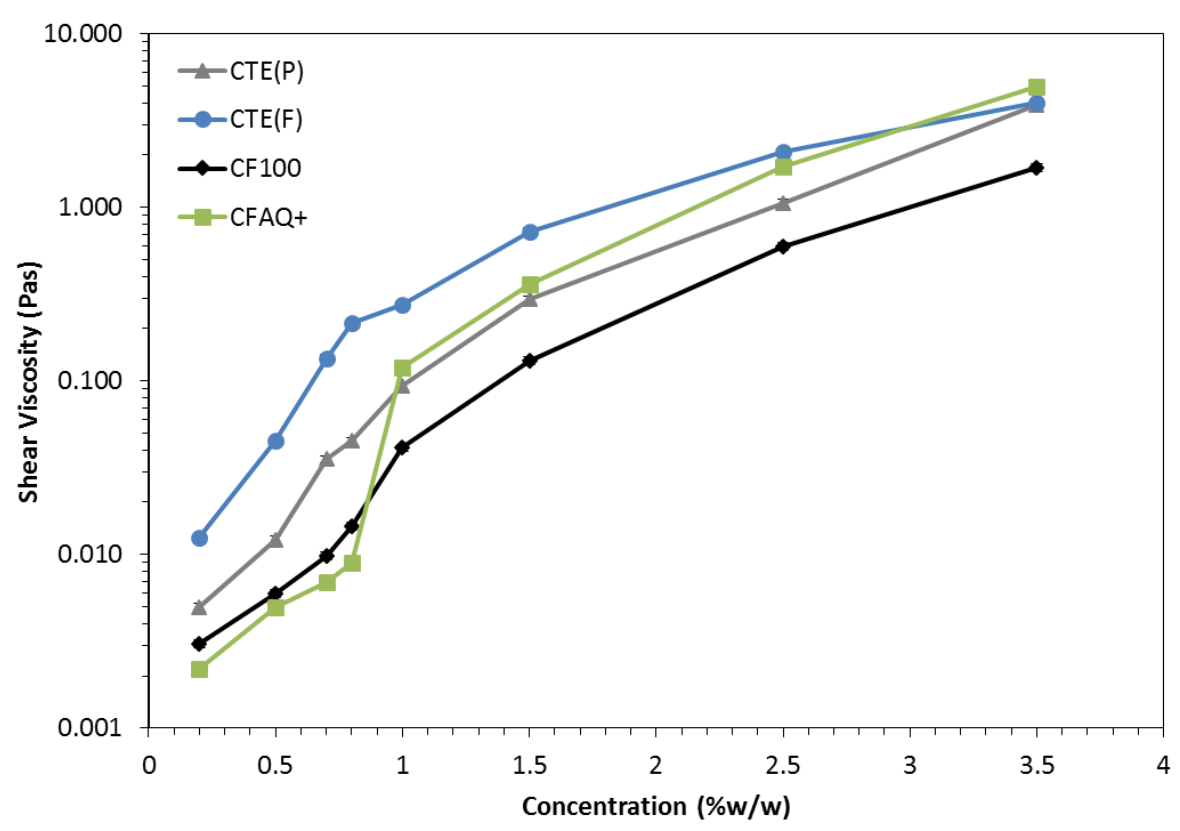

Figure 4: Concentration dependence of shear viscosity (Pas) recorded at shear rate $50 \mathrm{~s}^{-1}$ for

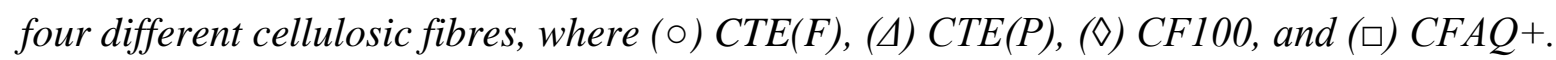

\subsubsection{Concentration dependence of shear viscosities:}

Shear viscosity recorded at a shear rate of $50 \mathrm{~s}^{-1}$ as a function of concentration is presented in Figure 4, and again shows the trend $\mathrm{CTE}(\mathrm{F})>\mathrm{CFAQ}+>\mathrm{CTE}(\mathrm{P})>\mathrm{CF} 100$, indicating that the shear viscosity is dependent on the source, processing and microstructure of cellulosic fibres. At the highest concentration studied $(3.5 \% \mathrm{w} / \mathrm{w})$ the CFAQ+ showed a higher viscosity than CTE(F), indicating that when the dispersions become highly packed the insoluble particles of CFAQ+ become dominant in the measured viscosity outcome, and the entangled nature of the $\mathrm{CTE}(\mathrm{F})$ is less effective at providing a measured viscosity. From a colloidal point of view, this then may be considered as an effect of 'hard' versus 'soft' and deformable particles. However, for some food applications such as ice-cream, mayonnaise, salad dressings etc., a maximum concentration of $0.8 \% \mathrm{w} / \mathrm{w}$ is recommended for citrus fibres considering the sensory perception without any off-flavour, body and texture defects [13]. Considering the maximum concentration $0.8 \% \mathrm{w} / \mathrm{w}$ for certain application, $\mathrm{CTE}(\mathrm{F})$ showed higher shear viscosity as compared to other cellulosic fibres (Figure 4). These results indicate that to achieve specific 
target viscosity (in the range of normal liquid-like foods) a lower concentration of $\mathrm{CTE}(\mathrm{F})$ is required as compared to other fibres(Figure 4 and Table 1). Such results of being able to match the viscosity of the different fibres in model systems can be considered for the purpose of investigating whether the inherent properties of the materials themselves can have an impact on sensory characteristics of texture and taste perception analysis.

\subsection{Sensory perception}

Figure 5 summarises the results from the sensory panel at the same concentration and matching viscosities (low and high viscosity) of four cellulosic fibres. In figure 5a, no significant differences ( $\mathrm{p}$-value $>0.05$ ) was found in saltiness perception of the four product at the same concentration $(1.5 \% \mathrm{w} / \mathrm{w})$. This can be attributed to the fact that at this concentration, where differences in moduli and WRV were seen, all measured viscosities, at $50 \mathrm{~s}^{-1}$, were $>500 \mathrm{mPas}$. This is significantly higher than the viscosity known to be important for decreasing the taste perception in entangled polymeric systems $[29,30]$, above critical concentration $\left(\mathrm{c}^{*}\right)$, and therefore at these higher viscosities, the effect of the fibre type is not apparent. Figuerola (2005) showed that texture was strongly dependent on the particle size in the case of citrus fibres [47]. Due to a noticeable difference in the microstructure of all four fibres at the same concentration, as expected, panellists reported $\mathrm{CTE}(\mathrm{F})$ suspensions were much thicker as compared to other cellulosic fibres suspensions (results not shown). 
(a)

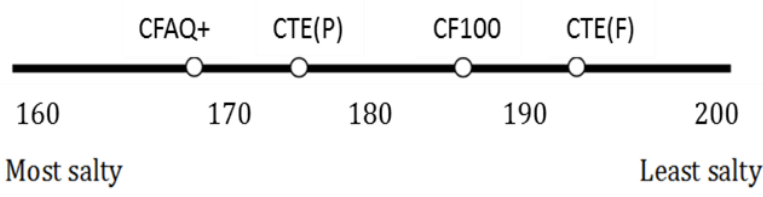

322

(b)

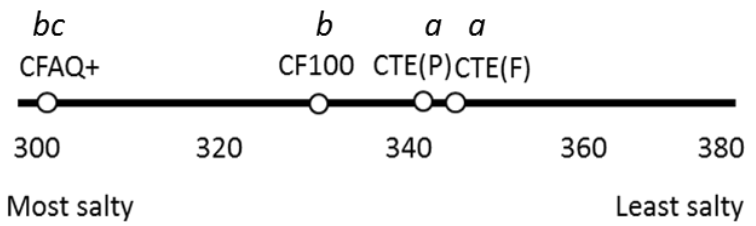

(c)

$\begin{array}{llll}b c & b & a & a\end{array}$

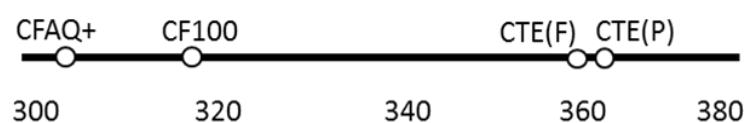

Most salty

Least salty

Figure 5: Rank sum scores of each sample $(C F A Q+, C F 100$ and $C T E(F)$ and $C T E(P))$ for saltiness perception, where a decreasing numerical value corresponds to an increase in the attribute. (a) At constant concentration i.e. $1.5 \%$ w/w, (b) At matched high viscosity (0.2Pas), and (c) At matched low viscosity (0.01Pas) salt suspensions [a, b, c represents the statistical significance, where the same letter indicates no significant difference, different letters indicate a significant difference with $p$-value $<0.05$. Note: $* b c$ indicates that no significant difference between CF100 and CFAQ+, but there is a significant difference (p-value<0.001) between $C F A Q+$ and CTE fibres.

It was evident from the rheological analysis in Figure 4, that an aqueous suspension of CTE(F) shows higher shear viscosities and this difference in shear viscosity explains the difference in thickness perception noted by the panellists during sensory analysis of $1.5 \% \mathrm{w} / \mathrm{w}$ suspension of different fibres. In the comments section, most of the panellists found a strong off-taste (described as 'citrus/lemon taste') with CF100 and little off-taste with CFAQ+ suspensions.

340 Whereas the absence of such off-taste was reported by the panellists (evident with no-

341 comments from the panels and even some panel mentioned no-off-taste when comparing with

$342 \mathrm{CF} 100$ and $\mathrm{CFAQ}+)$ in the case of $\mathrm{CTE}(\mathrm{F})$ and $\mathrm{CTE}(\mathrm{P})$ suspensions. 
343 In order to remove the impact of the difference in viscosities at the same concentration,

344 different suspensions were formulated with matched viscosities (low and high) but different

345 fibre concentrations. Using the paired comparison test, it was found that at high viscosity, a

346 significant reduction ( $\mathrm{p}$-value $<0.05$ ) in saltiness perception was observed with $\mathrm{CTE}(\mathrm{F})$ and

$347 \mathrm{CTE}(\mathrm{P})$ suspensions as compared to $\mathrm{CF} 100$ and CFAQ+ (Figure 5b). No significant difference

348 in terms of saltiness perception was observed between CTE(F) and CTE(P) as p-value>0.05.

349 Similar taste perceptions were observed with the suspensions at lower viscosities of different

350 cellulosic fibres (Figure 5c). The granular suspension of CF100 and CFAQ+ is believed to be

351 the cause of their higher saltiness perception, in line with similar behaviour found in particulate

352 suspensions such as starch and xanthan, in that if the granular structure was maintained during

353 processing, the system does not reduce the taste perception at high concentration [33, 34, 48].

354 A significant difference in saltiness perception and thickness between CTE products and citrus fibres (CFAQ+ and CF100) can be explained by the dense network structure afforded by the fibrillated cellulose - now acting more like a polymeric solution, resulting in reduced taste perception. While the differences in taste perception were significant between the CTE samples and the citrus samples, for both high and low viscosity, the positioning on the rank sum scoring for the higher viscosity systems was narrower. This then also indicates that all systems at the higher viscosities tend to behave as a concentrated dispersion, exemplified at the higher viscosities seen for the $1.5 \% \mathrm{w} / \mathrm{w}$ samples, where there was no difference seen between samples. In summary, it was evident from the sensory analysis, that the CTE samples with highly entangled network structure lowers the taste perception as compared to particulate suspensions such as CF100 and CFAQ+.

\section{Conclusions}

The influence of a highly entangled fibre network of cellulosic fibres on the rheological properties of a suspension is consistent with water retention values of these fibres and a key 
factor which may responsible for lower taste perception. Rheological measurements show that all cellulosic suspensions showed viscoelastic gel-like behaviour, due to highly dense fibrillar and particulate networks affording high water retention capacity. The difference in microstructures and inherent composition of different cellulosic fibres are responsible for difference in sensory (tastant) perception. Aqueous salt suspension at matched viscosities of softwood cellulosic fibre samples showed lower saltiness perception as compared to citrus fibres. It appears that the particulate structure releases the tastant more effectively and faster as compared to highly fibrillated and networked systems. The results presented in this paper clearly highlights that the choice of dietary fibre needs to be made carefully when considering the application in food products. A highly entangled network microstructure of cellulosic fibres, responsible for higher water retention capacity greater rheological properties may be beneficial for certain structural and nutritional aspects of food products, but if taste release is of importance, for increase sensory perception, then a fibrillated fibre would not be preferred over a more particulate material.

\section{Conflict of Interest}

There are no conflicts of interest to declare.

\section{Acknowledgement}

This work was supported by the Oslofjordfond, Norway grant scholarship (2012 - 2015). Special thanks to the Sensory Science Centre (the University of Nottingham) and Wenting Yin for help in running sensory sessions and all participants for their contribution.

\section{References}

[1] T.J. Foster. Technofunctionality of hydrocolloids and their impact on food structure. Gums and stabilisers for the food industry 15, Williams, P.A., and Phillips, G.O. (Eds). RSC: Cambridge, 2010, 103-112. 
392 [2] V. Tolstoguzov. Some thermodynamic considerations in food formulation. Food 393 Hydrocolloids. 2003, 17:1, 1-23.

394 [3] J. L. Kokini, J. B. Kadane and E. L. Cussler. Liquid texture perceived in mouth. Journal of 395 Texture Studies. 1977, 8:2, 195-218.

396 [4] C. M. Christensen. Oral perception of solution viscosity. Journal of Texture Studies. 1979, 397 $10: 2,153-164$.

398

[5] B.C. Tungland, D. Meyer. Non-digestible oligo- and polysaccharides (dietary fibre): their 399 physiology and role in human health and food. Comprehensive Reviews in Food Science and 400 Food Safety. 2002, 1, 73-92.

401

[6] S. Gorinstein, Z. Zachwieja, M. Folta, H. Barton, J. Piotrowicz, M. Zember, M. Weisz, S. 402 Trakhtenberg, O. Martin-Belloso. Comparative content of dietary fibre, total phenolic, and 403 minerals in persimmons and apples. Journal of Agricultural and Food Chemistry. 2001. 49, 404 952-957.

405

[7] J. Slavin Impact of the proposed definition of dietary fibre on nutrient databases. Journal of 406 Food Composition and Analysis. 2003, 16, 287-291.

407 [8] Herbafood. (2002). Herbacel AQ Plus. Apple and citrus fibre. Available from 408 www.herbafood.de/eaqplus.pdf.

409 [9] J. W. Anderson, B. M. Smith, N. S. Guftanson. Health benefit and practical aspects of high410 fibre diets. American Journal of Clinical Nutrition. 1994, 595, 1242-1247.

411 [10] Fiberstar. (2005). The product portfolio of Fiberstar Inc., US. Available at 412 http://fiberstar.net/

[11] M.A. Hill, J.R. Mitchell, P.A. Sherman. The relationship between the rheological and sensory properties of a lemon pie filling. Journal of Texture Studies. 1995, 26, 457-470. particle size and texture of mayonnaise. Food Science and Technology Research. 2007, 13:1, $417 \quad 1-6$. sensory properties of ice-cream. Food Science Technology International. 2006, 12:2,159-164. 
[14] H-U. Endress, J. Fischer. Fibres and fibre blends for individual needs: a physiological and technological approach. Advanced Dietary fibre technology. 2001, 26, 283-297.

422 [15] M. Pääkkö, M. Ankerfors, H. Kosonen, A. Nykänen, S. Ahola, M. Österberg, J. 423 Ruokolainen, J. Laine, P.T. Larsson, O. Ikkala, T. Lindström. Enzymatic Hydrolysis Combined with Mechanical Shearing and High-Pressure Homogenization for Nano-scale Cellulose Fibrils and Strong Gels. Biomacromolecules. 2007, 8, 1934-1941.

[16] G. Agoda-Tandjawa, S. Durand, S. Berot, C. Blassel, C. Gaillard, C. Garnier, L.J

Doublier.. Rheological characterization of microfibrillated cellulose suspensions after freezing.

[17] A. Cordoba, M.D.M. Camacho, N.M. Navarrete. Rheological behaviour of an insoluble lemon fibre as affected by stirring, temperature, time and storage. Food and Bioprocess Technology. 2010, 5-3, 1083-1092.

[18] D. Tatsumi, S. Ishioka, T. Matsumoto. Effect of fibre concentration and axial ratio on the rheological properties of cellulose fibre suspensions. Journal of the Society of Rheology Japan. 2002, 30:1, 27-32.

[19] D. Tatsumi. Rheology of cellulose fibre disperse systems and cellulose solutions. Nihon Reoroji Gakkaishi. 2007, 35:5, 251-256.

[20] E. Cepeda, \& I. Collado. Rheology of tomato and wheat dietary fibres in water and in 438 suspensions of pimento puree. Journal of Food Engineering. 2014, 134, 67-73.

[21] A. Torbica, M. Belovic, J. Mastilovic, Z. Kevresan, M. Pestoric, D. Skrobot, T.D. 440 Hadnadev. Nutritional, rheological, and sensory evaluation of tomato ketchup with increased content of natural fibres made from fresh tomato pomace, Food and Bioproducts Processing. 2016, 98, 299-309.

[22] J. Ahmed, A.S. Almusallam, F. Al-Salman, M.H. Abdul Rahman, E. Al-Salem. 444 Rheological properties of water insoluble date fiber incorporated wheat flour dough. LWT 445 Food Science and Technology. 2013, 51:2, 409-416.

446 [23] N. R. Cook, J. A. Cutler, E. Obarzanek, J. E. Buring, K. M. Rexrode, S. K. Kumanyika, 447 L. J. Appel, P. K. Whelton. Long term effects of dietary sodium reduction on cardiovascular 448 disease outcomes: Observational follow-up of the trials of hypertension prevention (TOHP). 449 British Medical Journal. 2007, 334, 885-888. 
450 [24] F. M. Sacks, L. P. Svetkey, W.M. Vollmer, L. J. Appel, G. A. Bray, D. Harsha, E. 451 Obarzanek, P. R. Conlin, E. R. Miller, D.G. Simons-Morton, N. Karanja, P. H. Lin, M. Aickin, M. M. Most-Windhauser, T. J. Moore, M.A. Proschan, J. A. Cutler. Effects on blood pressure of reduced dietary sodium and the dietary approaches to stop hypertension (DASH) diet. The New England Journal of Medicine. 2001, 344, 3-10.

[25] X. Tian, I.D. Fisk. Salt release from potato crisp. Food and Function. 2012, 3, 376-380.

[26] E.J. Lynch, F.D. Bello, E.H. Sheehan, K.D. Cashman, E.K. Arendt. Fundamental studies on the reduction of salt on dough and bread characteristic. Food Research International. 2009, $42,885-891$.

[27] A. Sullo, R.L. Watson, I.T. Norton. The design of colloidal foods for healthier diets. Gums and Stabilisers for the food industry: Changing the face of food manufacture: the role of hydrocolloids -17. Royal society of chemistry, Cambridge, UK. 2014, 289-299.

[28] R. Rama, N. Chiu, M.C.D. Silva, L. Hewson, J. Hort, I.D. Fisk. The impact of salt crystal size on the in-mouth delivery of sodium and saltiness perception from snack foods. Journal of Texture Studies. 2013, 44, 338-345.

[29] Z. V. Baines, \& E. R. Morris. Flavour/taste perception in thickened systems: the effect of guar gum above and below c*. Food Hydrocolloids. 1987, 1:3, 197-205.

[30] D. J. Cook, T. A. Hollowood, R. S. T. Linforth, A. J. Taylor. Oral shear stress predicts flavour perception in viscous solutions. Chemical Senses. 2003, 28:1, 11-23.

[31] R.J. Hill. Elastic modulus of microfibrillar cellulose gels. Biomacromolecules. 2008, 9, 2963-2966.

[32] T. A. Hollowood, R. S. T. Linforth, A. J. Taylor. The effect of viscosity on the perception of flavour. Chemical Senses. 2002, 27:7, 583-591. flavour perception: why is starch different from hydrocolloids? Food Hydrocolloids. 2006, $20: 6,855-862$.

[34] R. Abson, S.R. Gaddipati, J. Hort, J.R. Mitchell, B. Wolf, S.E. Hill, A comparison of the sensory and rheology properties of molecular and particulate forms of xanthan gum. Food Hydrocolloids. 2014, 53, 85-90. 
[35] J.F. Thibault, M. Lahaye, F. Guillon. Physiochemical properties of food plant cell walls. In: T. F. Schweizer, C. A. Edwards (eds) Dietary fibre, a component of food. Nutritional function in health and disease. Springer-verlag, Berlin. 1992, 21-56.

[36] A. L. Nelson. Properties of high-fibre ingredients. Cereal Foods World. 2001, 46, 93-97.

[37] A. Sangnark, A. Noomhorm. Effect of particle sizes on functional properties of dietary fibre prepared from sugarcane bagasse. Food Chemistry. 2003, 80, 221-229.

[38] M. Elleuch, D. Bedigian, O. Roiseux, S. Besbes, C. Blecker, H. Attia. Dietary fibre and fibre-rich by-products of food processing: Characterisation, technological functionality and commercial applications: A review. Food Chemistry. 2011, 124, 411-421.

[39] N. Grigelmo-Miguel, O. Martina-Belloso. Characterization of dietary fibre from orange juice extraction. Food Research International. 1999a, 131, 355-361.

[40] J. Ubando, A. Navarro, M. A. Valdivia. Mexican lime peel: Comparative study on contents of dietary fibre and associated antioxidant activity. Food Chemistry. 2005, 89, 57-61.

[41] N. Vergara-Valencia, E. Granados-Pereza, E. Agama-Acevedo, J. Tovarb, J. Rualesc, L. A. Bello-Pereza. Fibre concentrate from mango fruit: Characterization, associated antioxidant capacity and application as a bakery product ingredient. Lebensmittel-Wissenschaft und Technologie. 2007, 40, 722-729.

[42] N. Grigelmo-Miguel, O. Martina-Belloso. Influence of fruit dietary fiber addition on physical and sensorial properties of strawberry jams. Journal of Food Engineering. 1999b, 41, $13-21$.

[43] V. S. Eim, S. Simal, C. Rosselló, A. Femenia. Effects of addition of carrot dietary fibre on the ripening process of a dry fermented sausage (sobrassada). Meat Science. 2008, 80, 173182.

[44] C. Collar, E. Santos, C. M. Rosell. Assessment of the rheological profile of fibre-enriched bread doughs by response surface methodology. Journal of Food Engineering. 2007, 78, 820826.

[45] C. M. Rosell, E. Santos, C. Collar. Mixing properties of fibre enriched wheat bread doughs: A response surface methodology study. European Food Research and Technology. 2006, 223, 333-340. 
508 [46] P. Chen, H. Yu, Y. Liu, W. Chen, X. Wang, M. Ouyang. Concentration effects on the 509 isolation and dynamic rheological behaviour of cellulose nanofibres via ultrasonic processing. 510 Cellulose. 2013, 20, 149-157.

511 [47] F. Fernando, M. LuzHurtado, A. M. Estévez, I. Chiffelle, F. Asenjo. Fibre concentrates 512 from apple pomace and citrus peel as potential fibre sources for food enrichment. Food 513 Chemistry. 2005, 91:3, 395-401.

514 [48] E.R. Morris. Rheological and organoleptic properties of food hydrocolloids K. Nishinari, 515 E. Doi (Eds.), Food hydrocolloids, Plenum Press, New York. 1993, 201-210. 